\title{
An interval number-based multiple attribute bid-decision making model for substation equipments
}

\author{
Lili Zhu ${ }^{\mathrm{a}}$, \\ State Grid Material Co., Ltd, 100120 Beijing, China
}

\begin{abstract}
By analyzing the characteristics of public bidding for substation equipments and combining with the research methods of multiple attribute decision-making problems, a multiple attribute bid-decision making model is presented. Firstly, the weight of interval numbers is specified by using the interval numbers theory and entropy theory. Secondly, the deviation degree of decision-making scheme is proposed. Then the schemes are sorted. A typical case is analyzed based on the above-mentioned.
\end{abstract}

\section{Introduction}

Multiple attribute decision making is to select optimal alternatives or to order schemes in consideration of multiple attributes, widely existing in engineering ${ }^{[1-2]}$, economic, social ${ }^{[3]}$ and daily life. The complexity and the fuzziness of the objective things makes the multiple attribute decision problems encountered mostly uncertain and fuzzy. The uncertain multi-attribute decision making as a hot research topic has already caused extensive attention of scholars.

Equipment bidding is an effective means to improve the quality of equipment procurement and reduce the purchasing cost. With the rapid development of the power grid, especially the large-scale construction of UHV power grid, the investment to substation equipments has increased correspondingly. Meanwhile, the substation equipment investment has some characteristics, such as large amount and high risk. The effective bidding methods to achieve efficient investment have become a major problem which needs to consider.

The uncertainty is mostly discussed with triangular fuzzy numbers, trapezoidal fuzzy numbers and interval numbers. This paper aims to establish a substation equipment bidding model with multiple attribute decision making theory based on interval numbers. The model ultimately helps to ensure appropriate substation equipment purchase strategy and to ensure the power grid projects carried out smoothly.

\section{Substation \\ equipment bidding characteristics}

According to the difference of the subject matter, the bidding can be divided into engineering bidding, equipment bidding, material bidding and supervision bidding, etc. Equipment bidding can be divided into two types as general equipments and large-scale industrial equipments according to the generality and the level of standardization. The substation primary equipment because of its special purpose and huge investment obviously does not belong to general equipment, then it as a large-scale equipment bidding has the following characteristics.

(1) The contract features: the subject matter is of large amount, and the rights and obligations of the contract is from the beginning of the purchasing, until the equipment production and operation to the expiration of the warranty period.

(2) The equipment characteristics: The risk of the equipment out of order is huge. Thus, the reliability requirement of the substation equipments may be higher and the agreed quality is more complex.

(3) The requirements of the bidder's ability: the bidders are of higher qualification and ability requirements.

According to the substation equipment bidding process, the bidders offer the technology response and the business documents simultaneously.

\section{Traditional equipment bidding method}

Next, we study the method of selecting the final winning bidder after the bidders submit the response documents. In practice, two common methods are the lowest price method and the comprehensive evaluation method. The lowest price method applies to equipments the quality of which can be compared easily, and the price can be considered as the only factor to select the winning bidder, such as simple equipment, semi-finished products, general standard equipment and raw materials. And the bidder with the lowest price is the winning. Comprehensive evaluation method is to carry out the overall evaluation and comparison of the response documents considering the price standard and non price standard. With the comprehensive evaluation method, the non-price factors are folded into price standard and are given corresponding weighted in order to determine the

\footnotetext{
a Corresponding author: lilizhu1223@yeah.net
} 
lowest bidding price. The winning bidder evaluated as the lowest bid or the best bid is recognized as the best comprehensive evaluation of the bid.

For the power grid project, in the case of the substation equipment bidding, the main consideration is not the price but the special technology, quality assurance and compliance capabilities, etc., so the comprehensive evaluation method is generally used. However, the following reasons will lead to unfair using this method. Firstly, it is difficult to achieve the accuracy of the evaluation items and their quantitative standards. In practice, many evaluation factors are difficult to quantify, and the quantitative given value is not able to reflect the actual situation of bidders. In addition, the overall level of knowledge and subjective tendency will seriously restrict the accuracy and effectiveness of evaluation from judges. So it is necessary to use some mathematical tools for the bid-evaluation, in order to weaken the subjective will and to strengthen the accuracy and scientific of the evaluation. Existing several mathematical evaluation methods are based on fuzzy theory, neural network and real options theory and interval number theory.

\section{Bid-evaluation model of interval number-based multi-attribute decision making}

According to the multiple attribute decision making theory and traditional entropy theory, the weight of interval numbers is specified. Then, the deviation degree of decision-making scheme is proposed. Finally, the schemes are sorted ${ }^{[4-6]}$.

Suppose an uncertain multiple attribute decision making set as $A=\left\{A_{1}, A_{2}, \ldots, A_{n}\right\}$, an attribute set as $G=\left\{G_{1}, G_{2}, \ldots, G_{\mathrm{m}}\right\}$. Measure the $i$-th scheme $A_{\mathrm{i}}$ according to the $j$-th attribute $G_{\mathrm{j}}$ as the attribute value $\tilde{a}_{i j}=\left[a_{i j}^{L}, a_{i j}^{U}\right]$.

Supposeing $I_{t}(\mathrm{t}=1,2)$ is the subscripts of benefit indexes and cost indexes respectively, and $M=\{1,2, \ldots, m\}$, $N=\{1,2, \ldots, n\}$.

\subsection{The entropy-based attribute weights}

The steps to caculate attribute weights based on entropy theory are as follows.

(1) Transform the decision matrix $A=\left(a_{i j}\right)_{n \times m}$ into the deviation degree matrix $Q=\left(q_{i j}\right)_{n \times m}$, where

$$
q_{i j}=\mathrm{D}\left(a_{i j}, a_{j}^{*}\right)
$$

and $a_{j}^{*}=\left[a_{j}^{-}, a_{j}^{+}\right]$, where

$$
\begin{aligned}
& a_{j}^{-}=\max _{i} a_{i j}^{-}, a_{j}^{+}=\max _{i} a_{i j}^{+}, \text {for } j \in I_{l}, \\
& a_{j}^{-}=\min _{i} a_{i j}^{-}, a_{j}^{+}=\min _{i} a_{i j}^{+}, \text {for } j \in I_{2} .
\end{aligned}
$$

(2) Normalize the deviation degree matrix $Q=\left(q_{i j}\right)_{n \times m}$ as matrix $P=\left(p_{i j}\right)_{n \times m}$, where

$$
p_{i j}=\frac{q_{i j}}{\sum_{i=1}^{n} q_{i j}} .
$$

Obviously,

$$
\sum_{i=1}^{n} p_{i j}=1, j \in M
$$

(3) Caculate the entropy of attribute $G_{j}$ as

$$
S_{\mathrm{j}}=-k \sum_{i=1}^{n} p_{i j} \ln p_{\mathrm{ij}}, j \in M,
$$

where $k$ is a constant and $k>0$, ln denotes the natural logarithm, and

$$
S_{j} \geq 0, j \in M
$$

If $p_{\mathrm{ij}}=1 / n, i \in N, S_{j}$ gets the maximum, i.e.

$$
S_{j}=k \ln n, \text { and } 0 \leq S_{j} \leq 1 .
$$

(4) Caculate the deviation degree coefficient $u_{j}$ of each decision-making scheme for attribute $G_{j}$, and

$$
u_{j}=1-S_{j}, j \in M
$$

(5) Calculate the weight $\omega_{j}$ of each attribute, and

$$
\omega_{j}=\frac{u_{j}}{\sum_{j=1}^{m} u_{j}}, j \in M .
$$

\subsection{The deviation degree-based sorting method}

The steps to sort the decision-making schemes based on deviation degree of the interval numbers are putforwarded as follows.

(1) Constructing matrix $C=\left(c_{i j}\right)_{n \times m}$ and normalize it as matrix $R=\left(r_{i j}\right)_{n \times m}$ according to the evaluation target type, that is

$$
\begin{gathered}
c_{i j}=\frac{a_{i j}}{\max _{i} a_{i j}}=\left[\frac{a_{i j}^{-}}{\max _{i} a_{i j}^{+}}, \frac{a_{i j}^{+}}{\max _{i} a_{i j}^{-}}\right]=\left[c_{i j}^{-}, c_{i j}^{+}\right] i \in N, j \in I_{I} \\
c_{i j}=\frac{\min _{i} a_{i j}}{a_{i j}}=\left[\frac{\min _{i} a_{i j}^{-}}{a_{i j}^{+}}, \frac{\min _{i} a_{i j}^{+}}{a_{i j}^{-}}\right]=\left[c_{i j}^{-}, c_{i j}^{+}\right] \quad i \in N, j \in I_{2}
\end{gathered}
$$

and

$$
r_{i j}=\frac{c_{i j}}{\max _{i} c_{i j}^{+}}=\left[r_{i j}^{-}, r_{i j}^{+}\right] .
$$

(2) Considering the weighted vector $\omega$, construct bilaterally augmented matrix $B$, which is weighted and normalized, and 


$$
\begin{gathered}
G_{1} \\
B= \\
A_{1} \\
\vdots \\
A_{n} \\
A^{*} \\
A^{-}
\end{gathered}\left[\begin{array}{ccc}
\omega_{1} r_{11} & \cdots & \omega_{m} \\
\vdots & & \vdots \\
\omega_{1} r_{n 1} & \cdots & \omega_{m} r_{n m} \\
\omega_{1} r_{1}^{*} & \cdots & \omega_{m} r_{m}^{*} \\
\omega_{1} r_{1}^{-} & \cdots & \omega_{m} r_{m}^{-}
\end{array}\right],
$$

$r_{j}^{*}=\left[\left(r_{j}^{*}\right)^{-},\left(r_{j}^{*}\right)^{+}\right]$is the ideal attribute value of attribute $G_{\mathrm{j}}$, and

$$
\left(r_{j}^{*}\right)^{-}=\max _{i} r_{i j}^{-},\left(r_{j}^{*}\right)^{+}=1 .
$$

The scheme constructed from the ideal attribute value is the ideal scheme, denoted as $A^{*}$.

$r_{j}^{-}=\left[\left(r_{j}^{-}\right)^{-},\left(r_{j}^{-}\right)^{+}\right]$is the negative ideal attribute value of attribute $G_{\mathrm{j}}$, and

$$
\left(r_{j}^{-}\right)^{-}=\min _{i} r_{i j}^{-},\left(r_{j}^{-}\right)^{+}=\min _{i} r_{i j}^{+} .
$$

The scheme constructed from the negative ideal attribute value is the negative ideal scheme, denoted as $A^{-}$.

(3) Calculate the total deviation degree $d_{i}^{*}$ of scheme $A_{i}$ to the ideal scheme $A^{*}$, and the total deviation degree $d_{i}^{-}$of scheme $A_{i}$ to the ideal scheme $A^{-}, i \in N$, and

$$
\begin{aligned}
& d_{i}^{*}=\sum_{j=1}^{m} D\left(\omega_{j} r_{i j}, \omega_{j} r_{j}^{*}\right), i \in N, \\
& d_{i}^{-}=\sum_{j=1}^{m} D\left(\omega_{j} r_{i j}, \omega_{j} r_{j}^{-}\right), i \in N .
\end{aligned}
$$

(4) Calculate the relative closeness $d_{i}$ of scheme $A_{i}$ to the ideal scheme $A^{*}, i \in N$, and

$$
d_{i}=\left\{\begin{array}{ll}
\frac{d_{i}^{-}}{d_{i}^{*}} & d_{i}^{*} \neq 0 \\
+\infty & d_{i}^{*}=0
\end{array} .\right.
$$

(5) Sort the corresponding scheme $A_{i}$ according to the size of the relative closeness $d_{i}$.

And if $d_{p}>d_{q}, A_{p} \succ A_{q}$, for $p, q \in N$.

\section{Application in bid-evaluation for substation equipment}

Taking $A$ project as an example, a new substation equipment is needed for the power grid project. A total of 5 bidders $\left(A_{1}, A_{2}, A_{3}, A_{4}, A_{5}\right)$ participate in the bidding. The main factors considered in the bid-evaluation are economy, maintainability and reliability. Divided the factors into five attributes as fixed cost $G_{l}$, operating cost $G_{2}$, noise $G_{3}$, maintainability $G_{4}$ and reliability $G_{5}$. $G_{l}, G_{2}$ and $G_{3}$ are cost indexes, and $G_{4}$ and $G_{5}$ are benefit indexes. The original decision-making matrix based on interval number is listed in Table 1 .
Table 1. Interval number decision-making matrix $A$

\begin{tabular}{|c|c|c|c|c|c|}
\hline & $G_{I}$ & $G_{2}$ & $G_{3}$ & $G_{4}$ & $G_{5}$ \\
\hline$A_{I}$ & {$[3.7,4]$} & {$[5.9,6.8]$} & {$[30,40]$} & {$[3,5]$} & {$[90,100]$} \\
\hline$A_{2}$ & {$[2.5,3]$} & {$[4.7,5.7]$} & {$[66,75]$} & {$[3,5]$} & {$[70,80]$} \\
\hline$A_{3}$ & {$[3.5,4.5]$} & {$[4.6,5.4]$} & {$[35,45]$} & {$[8,10]$} & {$[75,85]$} \\
\hline$A_{4}$ & {$[4.2,4.8]$} & {$[4.8,5.9]$} & {$[35,48]$} & {$[8,12]$} & {$[95,98]$} \\
\hline$A_{5}$ & {$[3.6,4.2]$} & {$[5.6,6.9]$} & {$[68,75]$} & {$[4,7]$} & {$[76,86]$} \\
\hline
\end{tabular}

\subsection{Calculation of entropy-based attribute weights}

According to the steps of the model, the attribute weights are calculated firstly.

The deviation degree matrix $Q$ is obtained in Table 2 .

Table 2. The deviation degree matrix $Q$

\begin{tabular}{|c|c|c|c|c|c|}
\hline & $G_{1}$ & $G_{2}$ & $G_{3}$ & $G_{4}$ & $G_{5}$ \\
\hline$A_{1}$ & 1.56 & 1.91 & 0 & 8.6 & 5 \\
\hline$A_{2}$ & 0 & 0.32 & 50.21 & 8.6 & 32.02 \\
\hline$A_{3}$ & 1.8 & 0 & 7.07 & 2 & 20.62 \\
\hline$A_{4}$ & 2.48 & 0.54 & 9.43 & 0 & 2 \\
\hline$A_{5}$ & 1.63 & 1.8 & 51.66 & 6.4 & 23.6 \\
\hline
\end{tabular}

Normalize the deviation degree matrix, the matrix $\mathrm{P}$ is obtained in Table 3.

Table 3. The normalized deviation degree matrix $P$

\begin{tabular}{|c|c|c|c|c|c|}
\hline & $G_{I}$ & $G_{2}$ & $G_{3}$ & $G_{4}$ & $G_{5}$ \\
\hline$A_{I}$ & 0.21 & 0.42 & 0 & 0.34 & 0.06 \\
\hline$A_{2}$ & 0 & 0.07 & 0.42 & 0.34 & 0.38 \\
\hline$A_{3}$ & 0.24 & 0 & 0.06 & 0.08 & 0.25 \\
\hline$A_{4}$ & 0.33 & 0.12 & 0.08 & 0 & 0.02 \\
\hline$A_{5}$ & 0.22 & 0.39 & 0.44 & 0.25 & 0.28 \\
\hline
\end{tabular}

Caculate the entropy of attribute $S_{\mathrm{j}}$ as

$S_{1}=0.85, S_{2}=0.73, S_{3}=0.68$,

$S_{4}=0.8, S_{5}=0.83$.

Caculate deviation degree coefficient $u_{j}$ as

$u_{1}=0.15, u_{2}=0.27, u_{3}=0.32$, 
$u_{4}=0.2, u_{5}=0.17$.

Calculate the weight $\omega_{j}$ as

$\omega_{1}=0.14, \omega_{2}=0.24, \omega_{3}=0.29$,

$\omega_{4}=0.18, \omega_{5}=0.15$.

\subsection{Sort of the schemes deviation degree-based}

The normalized matrix $R$ can be obtained in Table 4 as follows.

Table 4. The normalized interval number matrix $R$

\begin{tabular}{|c|c|c|c|c|c|}
\hline & $G_{1}$ & $G_{2}$ & $G_{3}$ & $G_{4}$ & $G_{5}$ \\
\hline$A_{1}$ & $\begin{array}{c}{[0.53} \\
0.68]\end{array}$ & $\begin{array}{c}{[0.58,} \\
0.79]\end{array}$ & {$[0.56,1]$} & $\begin{array}{l}{[0.17,} \\
0.42]\end{array}$ & {$[0.86,1]$} \\
\hline$A_{2}$ & {$[0.69,1]$} & $\begin{array}{c}0.69, \\
0.98]\end{array}$ & $\begin{array}{l}{[0.3,} \\
0.46]\end{array}$ & $\begin{array}{c}0.17, \\
0.42]\end{array}$ & $\begin{array}{c}0.67 \\
0.8]\end{array}$ \\
\hline$A_{3}$ & $\begin{array}{c}0.47, \\
0.72]\end{array}$ & {$[0.73,1]$} & $\begin{array}{l}{[0.5,} \\
0.86]\end{array}$ & $\begin{array}{c}{[0.45,} \\
0.83]\end{array}$ & $\begin{array}{c}0.71, \\
0.95]\end{array}$ \\
\hline$A_{4}$ & $\begin{array}{c}0.43, \\
0.59]\end{array}$ & $\begin{array}{c}0.67, \\
0.97]\end{array}$ & $\begin{array}{c}0.47, \\
0.86]\end{array}$ & {$[0.45,1]$} & $\begin{array}{l}{[0.9} \\
0.98]\end{array}$ \\
\hline$A_{5}$ & $\begin{array}{l}{[0.5,} \\
0.69]\end{array}$ & $\begin{array}{c}0.57, \\
0.82]\end{array}$ & $\begin{array}{l}{[0.3,} \\
0.44]\end{array}$ & $\begin{array}{c}{[0.22,} \\
0.59]\end{array}$ & $\begin{array}{c}{[0.72,} \\
0.87]\end{array}$ \\
\hline
\end{tabular}

The ideal scheme $A^{*}$ and the negative ideal scheme $A^{-}$can be denoted as follows in Table 5 .

Table 5. The ideal and negative ideal schemes

\begin{tabular}{|c|c|c|c|c|c|}
\hline & $G_{1}$ & $G_{2}$ & $G_{3}$ & $G_{4}$ & $G_{5}$ \\
\hline$A^{*}$ & {$[0.69,1]$} & {$[0.73,1]$} & {$[0.56,1]$} & {$[0.45,1]$} & {$[0.9,1]$} \\
\hline$A^{-}$ & {$[0.43$,} & {$[0.57$,} & {$[0.3$,} & {$[0.17$,} & {$[0.67$,} \\
& $0.59]$ & $0.79]$ & $0.44]$ & $0.42]$ & $0.8]$ \\
\hline
\end{tabular}

Considering the weight $\omega_{j}$, construct and normalize the bilaterally augmented matrix $B$, listed in Table 6 as follows.

Table 6. The normalized bilaterally augmented matrix $B$

\begin{tabular}{|c|c|c|c|c|c|}
\hline & $G_{1}$ & $G_{2}$ & $G_{3}$ & $G_{4}$ & $G_{5}$ \\
\hline \multirow{2}{*}{$A_{1}$} & {$[0.07$,} & {$[0.14$,} & {$[0.16$,} & {$[0.03$,} & {$[0.13$,} \\
& $0.1]$ & $0.19]$ & $0.29]$ & $0.08]$ & $0.15]$ \\
\hline \multirow{2}{*}{$A_{2}$} & {$[0.1$,} & {$[0.17$,} & {$[0.09$,} & {$[0.03$,} & {$[0.1$,} \\
& $0.14]$ & $0.24]$ & $0.13]$ & $0.08]$ & $0.12]$ \\
\hline \multirow{2}{*}{$A_{3}$} & {$[0.07$,} & {$[0.18,0.24]$} & {$[0.15$,} & {$[0.08$,} & {$[0.11$,} \\
& $0.1]$ & & $0.25]$ & $0.15]$ & $0.14]$ \\
\hline \multirow{2}{*}{$A_{4}$} & {$[0.06$,} & {$[0.16$,} & {$[0.14$,} & {$[0.08$,} & {$[0.14$,} \\
& $0.08]$ & $0.23]$ & $0.25]$ & $0.18]$ & $0.15]$ \\
\hline \multirow{2}{*}{$A_{5}$} & {$[0.07$,} & {$[0.14,0.2]$} & {$[0.09$,} & {$[0.04$,} & {$[0.11$,} \\
& $0.1]$ & $0.13]$ & $0.11]$ & $0.13]$ \\
\hline \multirow{2}{*}{$A^{*}$} & {$[0.1$,} & {$[0.18$,} & {$[0.16$,} & {$[0.08$,} & {$[0.14$,} \\
& $0.14]$ & $0.24]$ & $0.29]$ & $0.18]$ & $0.15]$ \\
\hline \multirow{2}{*}{$A^{-}$} & {$[0.06$,} & {$[0.14$,} & {$[0.09$,} & {$[0.03$,} & {$[0.1$,} \\
& $0.08]$ & $0.19]$ & $0.13]$ & $0.08]$ & $0.12]$ \\
\hline
\end{tabular}

Calculate the total deviation degree $d_{i}^{*}$, and

$d_{1}^{*}=0.23, d_{2}^{*}=0.34, d_{3}^{*}=0.15$,

$d_{4}^{*}=0.13, d_{5}^{*}=0.4$.

Calculate the total deviation degree $d_{i}^{-}$, and

$d_{1}^{-}=0.23, d_{2}^{-}=0.13, d_{3}^{-}=0.32$,

$d_{4}^{-}=0.33, d_{5}^{-}=0.07$.

Calculate the relative closeness $d_{i}$, and

$d_{1}=1, d_{2}=0.38, d_{3}=2.13$,

$d_{4}=2.53, d_{5}=0.18$.

Then, the sorting results of the schemes is

$A_{4} \succ A_{3}, \succ A_{1} \succ, A_{2} \succ A_{5}$.

That is the scheme of bidder $A_{4}$ is optimal, and bidder $A_{4}$ is the winner of the bidding.

\section{Concluding remarks}

The interval number theory and entropy theory are applied to the substation equipment bid-evaluation multi attribute decision making problems in this paper. As its objective uncertainty analysis to the attributes, its helpful to weaken the subjective will and strengthen the accuracy and scientific of the bid-evaluation, .it has been proved that these theories for the procurement of substation equipments, not only greatly improve the efficiency of equipment procurement, but also greatly improve the benefit of equipment procurement. The model proposed in this paper is innovative method and thought innvation as well. The optimization of the model for the application of power grid equipment becomes the further research orientation.

\section{References}

1. L. Crhz, J. Mesa. Eur. J. Oper. Res.135(2011).

2. Y. Song. Syst. Eng. Theory Pract., 22(2002).

3. M. F. Shipley, A. Korvin, K. Omer. Eur. J. Oper. Res. 135(2011).

4. Z. Xu, Z. Le. J. Manage. Sci China. 5(2002).

5. Q Zhang. Syst. Eng. Theory Pract., 19(1999).

6. Q. Zhang, Z. Fan, D. Pan. Eng. Theory Pract., 19(2000). 